The object of the present note is to show clearly that $G$ may be regarded as a generalization of the dihedral group which includes the earlier generalization obtained by considering the groups generated by two operators which have a common square.* If two operators have a common square, this square is clearly invariant under the group generated by these operators; but if the squares are invariant under this group they evidently are not necessarily the same. From this it follows directly that the present generalization includes the earlier one, and it gives rise to an almost equally elementary category of groups as a result of the equations established in the preceding paragraph. If two operators have a common square, it is known that the product of either one into the inverse of the other is transformed into its inverse by each of the operators. The analogous theorem as regards the operators under consideration may be expressed as follows:

When each of two operators is commutative with the square of the other, the product of one into the inverse of the other is transformed by each of the two operators into its inverse multiplied by an invariant operator under the group generated by the two operators.

UNIVERSITY OF ILLINOIS.

\title{
THE SOLUTION OF THE EQUATION IN TWO REAL VARIABLES AT A POINT WHERE BOTH THE PARTIAL DERIVA- TIVES VANISH.
}

BY DR. L. S. DEDERICK.

(Read before the American Mathematical Society, September 14, 1909.)

If $F(x, y)$ is a real function of the real variables $x$ and $y$ which is continuous at and near the point $\left(x_{0}, y_{0}\right)$, and vanishes at this point, but has one first order partial derivative at the point not equal to zero, there are a number of well-known theorems about the existence of other values of $x$ and $y$ satisfying the equation

$$
F(x, y)=0,
$$

\footnotetext{
* Archiv der Mathematik und Physik, vol. 9 (1905), p. 6.
} 
and about the properties of $y$ as a function of $x$, or $x$ as a function of $y$, thus defined. The case, however, where both the first partial derivatives of $F(x, y)$ vanish at $\left(x_{0}, y_{0}\right)$, seems to have received very little attention, although analogous theorems for this case are tacitly used in most treatments of the singularities of plane curves. In this subject, of course, it is frequently assumed that $F(x, y)$ is analytic at the point in question; but even where much less than this is demanded, it frequently happens that more is required than is at all necessary,* that the exact conditions are not stated, and that existence proofs are omitted.

The object of the present paper is to examine the possible cases that may arise when the first partial derivatives both vanish, and to state theorems analogous to Dini's theorem in these cases. No attempt has been made to reduce the sufficient conditinns to an absolute minimum, but in most cases it seems unlikely that any simple statement could be made of conditions materially less restrictive. The general statement may be made concerning the requirements of continuity, that there must be one order of partial derivatives of $F(x, y)$ continuous at and near $\left(x_{0}, y_{0}\right)$ and not all vanishing at that point, and derivatives of any higher orders must be continuous at and near $\left(x_{0}, y_{0}\right)$ whose values at $\left(x_{0}, y_{0}\right)$ are required in order to determine the character of the solution or the value of a derivative of $y$ with respect to $x$ whose existence is asserted ; i. e., we may perform the formal differentiations for ascertaining any desired facts, and know that they are justified if all the partial derivatives of $F(x, y)$ that we use are continuous at and near $\left(x_{0}, y_{0}\right)$.

In all the following theorems we shall assume that $F(x, y)$, together with all its partial derivatives of order $n$ or lower, is continuous at and near $\left(x_{0}, y_{0}\right)$, and that $F(x, y)$ and all its derivatives of order less than n, vanish at this point, but that not all the derivatives of order $n$ are equal to zero there. The following notation will be used throughout:

$$
\begin{gathered}
h=x-x_{0}, \quad k=y-y_{0}, \quad \alpha=\frac{k}{h}=\frac{y-y_{0}}{x-x_{0}}, \\
F_{i j}(x, y)=\frac{\partial^{i+j} F(x, y)}{\partial x^{i} \partial y^{j}}, \quad F_{i j}=F_{i j}\left(x_{0}, y_{0}\right),
\end{gathered}
$$

\footnotetext{
*E. g., de la Vallée-Poussin, Cours d'analyse, vol. 2, chap. 8.
} 


$$
\begin{aligned}
& b_{i j}=\frac{1}{i ! j !}, \quad b F_{i j}=b_{i j} F_{i i}, \\
& \bar{F}_{i j}=F_{i j}\left(x_{0}+\theta h, y_{0}+\theta_{1} k\right) .
\end{aligned}
$$

where $0 \leqq \theta \leqq 1,0 \leqq \theta_{1} \leqq 1$. In most cases $0<\theta=\theta_{1}<1$, but not always. Moreover $\theta$ or $\theta_{1}$ need not preserve the same value throughout any one discussion.

THEOREM 1. - There is a neighborhood of $\left(x_{0}, y_{0}\right)$ in which all pairs of values of $x$ and $y$ satisfying equation (1) can be grouped into not more than $n$ branches such that on each branch $\alpha$ approaches a limit (finite or infinite) equal to some real root of the nth degree equation in $\alpha$

$$
\sum_{i=0}^{n} b F_{n-i, i} \alpha^{i}=0
$$

By Taylor's theorem for two variables there is a neighborhood of $\left(x_{0}, y_{0}\right)$ in which we can write

$$
F(x, y)=h^{n} \sum_{i=0}^{n} b \bar{F}_{n-i, i} \alpha^{i}
$$

If this vanishes when $x \neq x_{0}$, then

$$
\sum_{i=0}^{n} b \bar{F}_{n-i, i} \alpha^{i}=0
$$

From the continuity of the partial derivatives each coefficient in (4) approaches the corresponding coefficient in (2) as $(x, y)$ approaches $\left(x_{0}, y_{0}\right)$, and hence each root of (4) approaches some root of (2). There is, therefore, a neighborhood of $\left(x_{0}, y_{0}\right)$ in which the possible solutions of (1) are separated into not more than $n$ branches according to the root of (2) whose neighborhood contains the corresponding value of $\alpha$. If $F_{0, n}=0$, equation (2) must be regarded, of course, as having an infinite root; and accordingly, there may be a branch on which $\alpha$ becomes infinite. This includes the case where $F\left(x_{0}, y\right)=0$ throughout some interval including $y_{0}$. Here (3) is satisfied by the vanishing of $h$. In the subsequent theorems, however, we shall always understand by a root of equation (2) a real 
finite root. The question of a solution belonging to an infinite root is easily reduced to this by interchanging the variables $x$ and $y$.

By a branch here is meant merely a certain aggregate of pairs of values. We have not shown that a branch defines $y$ as a single valued function of $x$, nor yet a continuous function. We have shown, however, that the values of $x$ and $y$ belonging to one branch satisfy a relation

$$
y-y_{0}=\left(x-x_{0}\right)\left(\alpha_{1}+\zeta\right)=h\left(\alpha_{1}+\zeta\right),
$$

where $\alpha$ is a root of (2) and $\zeta$ approaches zero with $h$, and that in a sufficiently small neighborhood of $\left(x_{0}, y_{0}\right)$ any solution belongs to some one such branch.*

Theorem 2.-Corresponding to any simple root $\alpha_{1}$ of equation (2), there is a neighborhood $D$ of $\left(x_{0}, y_{0}\right)$ and $a$ single-valued function $f(x)$ such that when $y=f(x)$ in $D$, $F(x, y) \equiv 0$. This function is continuous and has a continuous derivative in $D$, and represents all the values of $x$ and $y$ in $D$ which satisfy (1) and belong to the branch corresponding to $\alpha_{1}$;

i. e., there is an interval $\left(\alpha_{1}-\gamma, \alpha_{1}+\gamma\right)$, such that if $(x, y)$ is in $D$, and $\alpha_{1}-\gamma<\alpha<\alpha_{1}+\gamma$, then $F(x, y)=0$ if and only if $y=f(x)$.

If $(x, y)$ and $\left(x_{1}, y_{1}\right)$ are any two points in the neighborhood of $\left(x_{0}, y_{0}\right)$,

$$
F(x, y)=F\left(x_{1}, y_{1}\right)+\left[F(x, y)-F\left(x_{1}, y_{1}\right)\right] .
$$

If $x=x_{1}$,

$F(x, y)-F\left(x_{1}, y_{1}\right)=F(x, y)-F\left(x, y_{1}\right)=\Delta y F_{01}\left(x, y_{1}+\theta \Delta y\right)$,

* Note. - For this theorem the conditions of continuity may be slightly reduced. It is sufficient to demand that the partial derivatives of $F(x, y)$ of order $n$ shall exist at and near $\left(x_{0}, y_{0}\right)$ and be continuous at that point. We can then stop the Taylor's development one term sooner and expand each partial derivative of order $(n-1)$ by the unsymmetrical form of the law of the mean,

$$
\begin{aligned}
\boldsymbol{F}_{n-1-i, i}\left(x_{0}+\theta h, y_{0}+\theta k\right) \\
=\theta h F_{n-i, i}\left(x_{0}+\theta_{1} \theta h, y_{0}\right)+\theta k F_{n-i-1, i+1}\left(x_{0}+\theta h, y_{0}+\theta_{2} \theta k\right) .
\end{aligned}
$$

This gives the same result as the Taylor's development except for the factor $n \theta$, which does not affect the reasoning. 
where $\Delta y=y-y_{1}$. In general,

In particular, let

$$
y=y_{0}+\alpha\left(x-x_{0}\right) \text {. }
$$

Then

$$
y_{1}=y_{0}+\alpha_{1}\left(x-x_{0}\right) \text {. }
$$

$$
\Delta y=y-y_{1}=\left(\alpha-\alpha_{1}\right)\left(x-x_{0}\right)=h \Delta \alpha,
$$

where $\Delta \alpha=\alpha-\alpha_{1}$. Hence

$$
F(x, y)=F\left(x, y_{1}\right)+h \Delta \alpha F_{01}\left(x, y_{1}+\theta \Delta y\right) .
$$

Now by Taylor's theorem,

(6) $F_{01}(x, y)=h^{n-1} \sum_{i=0}^{n-1} b_{n-1-i, i} \bar{F}_{n-1-i, i+1} \alpha^{i}=h^{n-1} \sum_{i=1}^{n} i b \bar{F}_{n-i, i} \alpha^{i-1}$.

As $h$ approaches zero and $\alpha$ approaches $\alpha_{1}$, the coefficient of $h^{n-1}$ in this expression approaches

$$
\sum_{i=1}^{n} i b F_{n-i, i} \alpha_{1}^{i-1}=M
$$

where $M \neq 0$ since $\alpha_{1}$ is a simple root of (2). If then we take any positive $\epsilon$ such that $\epsilon<|M|$, we can find a neighborhood $D_{1}$ of $\left(x_{0}, y_{0}\right)$ and an interval $\left(\alpha_{1}-\gamma, \alpha_{1}+\gamma\right)$, such that when $(x, y)$ is in $D_{1}$, and $\alpha_{1}-\gamma<\alpha<\alpha_{1}+\gamma$, we have

Again, from (3),

$$
\left|\sum_{i=1}^{n} i b F_{n-i, i}(x, y) \alpha^{i-1}\right|>\epsilon .
$$

$$
F\left(x, y_{1}\right)=h^{n} \sum_{i=0}^{n} b \bar{F}_{n-i, i} \alpha_{1}^{i}
$$

Since $\alpha_{1}$ is a root of (2), there is a neighborhood $D_{2}$ of $\left(x_{0}, y_{0}\right)$ in which

$$
\left|\sum_{i=0}^{n} b F_{n-i, i}(x, y) \alpha_{1}^{i}\right|<\epsilon|\Delta \alpha| .
$$

Substituting in (5) from (8) and (6), we have 


$$
F(x, y)=h^{n}\left[\sum_{i=0}^{n} b \bar{F}_{n-i, i} \alpha_{1}^{i}+\Delta \alpha \sum_{i=1}^{n} i b \bar{F}_{n-i, i} \alpha^{i-1}\right] .
$$

From $(7)$ and $(9)$, if $(x, y)$ lies in $D$, the region common to $D_{1}$ and $D_{2}$, and $\alpha_{1}-\gamma<\alpha<\alpha_{1}+\gamma$, then the first term in the bracket is less in absolute value that $\epsilon|\Delta \alpha|$, and the second is greater. If then we leave $h$ unchanged but replace $\Delta \alpha$ by its negative, we change the sign of $F^{\prime}(x, y)$. Therefore $F(x, y)$ must vanish between $y=y_{1}-\Delta y$ and $y=y_{1}+\Delta y$; and since in this interval $F_{01}(x, y)$ does not vanish, it remains of one sign, and hence $F(x, y)$ can not vanish more than once. Thus for any value of $x$ in $D$, there is one and only one value of $y$ between $y=y_{0}+h\left(\alpha_{1}-\gamma\right)$ and $y=y_{0}+h\left(\alpha_{1}+\gamma\right)$ for which $F(x, y)=0$. This defines $y$ as a single-valued function of $x$ in $D$, and makes it the complete solution which satisfies the condition $\lim _{x=x_{0}} \alpha=\alpha_{1}$. This last condition shows that $f(x)$ is continuous at $x=x_{0}$, and that $f^{\prime}\left(x_{0}\right)=\alpha_{1}$. When $y=f(x)$ in $D$ and $x \neq x_{0}$, we have seen that $F_{01}(x, y) \neq 0$. Hence by Dini's theorem, $f(x)$ and $f^{\prime}(x)$ are continuous in $D$ when $x \neq x_{0}$.

It only remains now to show that $f^{\prime}(x)$ is continuous at $x=x_{0}$. When $x \neq x_{0}$, we know that

$$
\begin{aligned}
f^{\prime}(x) & =-\frac{F_{10}(x, y)}{F_{01}(x, y)}=-\frac{h^{n-1} \sum_{i=0}^{n-1} b_{n-1-i, i} \bar{F}_{n-i, i} \alpha^{i}}{h^{n-1} \sum_{i=0}^{n-1} b_{n-1-i, i} \bar{F}_{n-1-i, i+1} \alpha^{i}} \\
& =-\frac{\sum_{i=0}^{n-1}(n-i) b \bar{F}_{n-i, i} \alpha^{i}}{\sum_{i=1}^{n} i b \bar{F}_{n-i, i} \alpha^{i-1}} .
\end{aligned}
$$

As $x$ approaches $x_{0}$ and hence $\alpha$ approaches $\alpha_{1}$, the numerator and denominator of this both approach limits, and we have seen that the limit of the latter is not zero. Therefore $\lim _{x=x_{0}} f^{\prime}(x)$ exists. But since $f(x)$ is continuous at $x_{0}$, this is sufficient that $f^{\prime}(x)$ be continuous there also.*

'This same general method of proof may be extended to the case where $\alpha_{1}$ is a multiple root. The method is essentially this : to determine an approximate solution and a strip includ-

* Dini, Funzioni di variabili reali, $\$ 75$; or E. W. Hobson, Theory of functions of a real variable, $\S 220$. 
ing it within which the actual solution must lie, and then to show that on the approximation $F(x, y)$ is so small, and throughout the strip $F_{01}^{\prime}(x, y)$ is so large, that for any value of $x, F(x, y)$ must vanish for one and only one value of $y$ in the strip. In Theorem 2, the approximation and the boundaries of the strip are straight lines. For later cases the approximation must be closer and the boundaries of the strip must approach each other more rapidly.

When $n=1$, this theorem reduces to Dini's theorem and the proof becomes that given by de la Vallée-Poussin (Cours d'analyse infinitésimale, I, \142). As in the case of Dini's theorem, we can show here that every additional order of partial derivatives of $F(x, y)$ which are continuous at and near $\left(x_{0}, y_{0}\right)$ insures the existence and continuity of an additional derivative of $f(x)$. This is shown in the following theorem, which completes the treatment of a simple root of equation (2).

Theorem 3. - If $f(x)$ is a solution of the sort discussed in Theorem 2, and the partial derivatives of $F(x, y)$ of order $(n+m)$ are continuous at and near $\left(x_{0}, y_{0}\right)$, then $f^{(m+1)}(x)$ exists and is continuous at and near $x_{0}$.

We know from Theorem 2 that this is true when $m=0$. To show that it is true in general, we assume that $f^{(m)}(x)$ exists and is continuous at and near $x_{0}$. This we know is true when $m=0$ or $m=1$. It is also true if this theorem holds for all values of $m$ less than the particular one in question. The proof, then, on the assumption that $f^{(m)}(x)$ is continuous, establishes the theorem by complete induction.

We can expand $F(x, y)$ by Taylor's theorem in powers of $h$ from $h^{n}$ to $h^{n+m}$ with coefficients that are polynomials in $\alpha$ and contain no other variable except in the case of the last. From the assumption of the continuity of $f^{(m)}(x)$, we can write when $y=f^{\prime}(x)$

$$
\alpha=f^{\prime}+\frac{1}{2} h f^{\prime \prime}+\cdots+\frac{1}{m !} h^{m-1}\left(f^{(m)}+\zeta\right),
$$

where $\zeta$ approaches zero with $h$. If we substitute this in the expansion of $F(x, y)$ and arrange according to powers of $h$, the coefficients of the powers up to and including $h^{n+m-2}$ will be constants. That of $h^{n+m-1}$ will contain $\zeta$ linearly but no other variable. Hence, since $F(x, y)=0$ when $y=f(x)$, we have an equation of the form,

$$
A_{n} h^{n}+\cdots+A_{n+m-2} h^{n+m-2}+(B+\omega \zeta) h^{n+m-1}+h^{n+m} \phi(h)=0,
$$


where $A_{n}, \cdots, A_{n+m-2}$, and $B$ are are constants, $\omega$ and $\phi$ approach limits as $h$ approaches zero, and

$$
\lim _{h=0} \omega=\frac{1}{m !} \sum_{i=1}^{n} i b F_{n-i, i} \alpha_{1}^{i} \neq 0 .
$$

Since $\zeta$ approaches zero with $h$,

and

$$
A_{n}=A_{n+1}=\cdots=A_{n+m-2}=B=0
$$

$$
\omega \zeta+h \phi(h)=0 .
$$

Therefore $\zeta=h \eta$, where $\lim _{h=0} \eta$ exists. If we put this in (10), we have a development of $\alpha$ including the power $h^{m}$, in which the coefficient of $h^{m-1}$ is constant. From the continuity of $f^{(m)}$, moreover, we have

$$
y^{\prime}=f^{\prime}(x)=f^{\prime}+h f^{\prime \prime}+\cdots+\frac{h^{m-1}}{(m-1) !}\left(f^{(m)}+\zeta_{1}\right),
$$

where $\zeta_{1}$ approaches zero with $h$. When $y=f(x)$ we have also

$$
F_{10}(x, y)+y^{\prime} F_{01}(x, y)=0 .
$$

If we expand the partial derivatives by Taylor's theorem and replace $\alpha$ and $y^{\prime}$ by their developments, we find that the powers of $h$ in the expansions of the partial derivatives run from $h^{n-1}$ to $h^{n+m-1}$, and hence after the substitution, the coefficients are constants up to and including that of $h^{n+m-3}$, while the coefficient of $h^{n+m-2}$ involves $\zeta_{1}$ linearly and no other variable. In the same way as for $\zeta$, we show that $\zeta_{1}=h \eta_{1}$ where $\lim _{h=0} \eta_{1}$ exists. This extends the development of $y^{\prime}$ one step further. In like manner, by substituting in the successive total derivatives of $F(x, y)$ with regard to $x$ and equating them to zero, we add one power of $h$ to the known developments of each of the derivatives, $y^{\prime \prime}, y^{\prime \prime \prime}, \ldots, y^{(m)}$, finally getting $y^{(m)}=f^{(m)}+h \eta_{m}$, where $\lim _{h=0} \eta_{m}$ exists. By Dini's theorem we know that when $x \neq x_{0}, y^{(m+1)}$ exists, and hence the $(m+1)$ th total derivative of $F(x, y)$ exists and is equal to zero. If we expand this in powers of $h$ and substitute for $\alpha, y^{\prime}, \cdots, y^{(m)}$ their developments in powers of $h$, we have an equation for determining $y^{(m+1)}$, in which the coefficient of $y^{(m+1)}$ is $F_{01}(x, y)$, or

$$
h^{n-1} \sum_{i=1}^{n} i b \bar{F}_{n-i, i} \alpha^{i} \text {. }
$$


In the rest of the equation the coefficients are constants up to and including that of $h^{n-2}$; and the coefficient of $h^{n-1}$ approaches a limit. We cannot infer here as before that the constant coefficients are all zero, because we do not know that $y^{(m+1)}$ approaches a limit as $h$ approaches zero. We can infer, however, that when $h$ approaches zero, $y^{(m+1)}$ either approaches a limit or else becomes infinite. But since $f^{(m+1)}(x)$ exists when $x \neq x_{0}$, we know that $y^{(m)}=f^{(m)}+h f^{(m+1)}\left(x_{0}+\theta h\right)$; and we have seen that $y^{(m)}=f^{(m)}+h \eta_{m}$. Therefore $f^{(m+1)}\left(x_{0}+\theta h\right)=\eta_{m}$, and this approaches a limit. But this is impossible if $f^{(m+1)}$ becomes infinite. Therefore $\lim _{x=x_{0}} f^{(m+1)}(x)$ exists and from this it follows that $J^{(m+1)}(x)$ is continuous at $x=x_{0}$.

There now remains to consider the case of a multiple root of equation (2). We know from the theory of plane curves that in this case the existence and character of the solution depend upon the values of derivatives of an order higher than $n$. The simplest case of this sort is treated in the following theorem.

THEOREM 4. - Let $F(x, y)$ have all its partial derivatives of order $(n+1)$ continuous at and near $\left(x_{0}, y_{0}\right)$, and let $\alpha_{1}$ be $a$ multiple root of order $m$ of equation (2); but let

$$
\sum_{i=0}^{n+1} b F_{n+1-i, i} \alpha_{1}^{i} \neq 0 \text {. }
$$

Then the values of $x$ and $y$ in the neighborhood of $\left(x_{0}, y_{0}\right)$ for which $F(x, y)=0$ and $\alpha$ approaches $\alpha_{1}$ are completely given by $a$ solution in the form $x=x_{0}+e t^{m}, y=y_{0}+e \alpha_{1} t^{m}+t^{m+1} f(t)$, where $e$ is +1 or -1 and $f(t)$ is a single-valued function which is continuous at $t=0$.

We shall omit the proof of this and proceed at once to a much more general discussion, from which this theorem follows as a special case. Let us assume that the partial derivatives of $F(x, y)$ of order $n+r$ are continuous at and near $\left(x_{0}, y_{0}\right)$. Then we can expand $F(x, y)$ in the form,

$$
F(x, y)=h^{n} \phi_{n}+h^{n+1} \phi_{n+1}+\cdots+h^{n+r} \bar{\phi}_{n+r},
$$

where $\phi_{i}$ is a polynomial of degree $i$ in $\alpha$, having for coefficients the partial derivatives of $F(x, y)$ of order $i$ with the arguments $\left(x_{0}, y_{0}\right)$ except where the dash as in $\bar{\phi}_{n+r}$ indicates the arguments $x_{0}+\theta h, y_{0}+\theta k$. Since the coefficients of $\bar{\phi}_{n+r}$ are continuous at and near $\left(x_{0}, y_{0}\right)$, we may write $\bar{\phi}_{n+r}=\phi_{n+r}+\zeta$, where $\zeta$ approaches zero with $h$. Then at any point of a solution 


$$
\phi_{n}+\phi_{n+1} h+\phi_{n+2} h^{2}+\cdots+\phi_{n+r} h^{r}+\zeta h^{r}=0 .
$$

We have seen in Theorem 1 that for any solution, $\alpha$ must approach a root of $\phi_{n}$, which we have called $\alpha_{1}$. In Theorems 2 and 3 we have discussed the case where $\alpha_{1}$ is a simple root. Theorem 4 supposes that $\alpha_{1}$ is a multiple root of $\phi_{n}$ but not a root of $\phi_{n+1}$. We shall now allow $\alpha_{1}$ to be a root of any number of $\phi$ 's and of any possible orders, and obtain certain necessary conditions for a solution.

Let $\alpha_{1}$ be a root of each of the polynomials, $\phi_{n}, \phi_{n+1}, \cdots, \phi_{n+r}$; and let its orders of multiplicity as a root of these be respectively $m_{0}, m_{1}, \cdots, m_{r}$. The important case that $\alpha_{1}$ is not a root of $\phi_{n+r}$ is included here by making $m_{r}=0$. We may assume without loss of generality that no previous $m$ is zero, as that case is treated by taking a smaller value of $r$. For any solution belonging to $\alpha_{1}$, we have $\alpha=\alpha_{1}+\zeta_{1}$ where $\zeta_{1}$ approaches zero with $h$. If we put this value of $\alpha$ in (12) and expand, the terms in $\zeta_{1}$ of degree less than $m_{i}$ in the expansion of $\phi_{i}$ will drop out from the vanishing of their coefficients, and we shall have

$$
\psi_{0}\left(\zeta_{1}\right) \zeta_{1}^{m_{0}}+\psi_{1}\left(\zeta_{1}\right) h \zeta_{1}^{m_{1}}+\cdots+\psi_{r}\left(\zeta_{1}\right) h^{r} \zeta_{1}^{m_{r}}+\zeta h^{r}=0
$$

where the $\psi$ 's are polynomials in $\zeta_{1}$ with constant coefficients, and the constant term in each is not zero. In sact

$$
\psi_{i}(0)=\frac{1}{m_{i} !}\left(\frac{\partial}{\partial \alpha}\right)^{m_{i}} \phi\left(\alpha_{1}\right) \neq 0 .
$$

Now let $c$ and $d$ be any two integers that are relatively prime, and let $e$ be +1 or -1 , its sign being at present undetermined. It is always possible, however, to choose its sign so that.for any value of $h$ there is at least one value of $t$ such that $h=e t^{d}$. If we define $t$ in this way set $\eta=\zeta_{1} / t^{c}$, we have $\zeta_{1}=\eta t^{c}$. Replacing $h$ and $\zeta_{1}$ in (13) by these values, we get

$$
\sum_{i=0}^{r} e^{i} \psi_{i}\left(\eta t^{c}\right) \eta^{m_{i}} t^{c m_{i}+d i}+\zeta e^{r} t^{d r}=0 .
$$

From this we may cancel out the lowest power of $t$ that appears and get an algebraic equation that $\eta$ must satisfy. If now we let $t$ approach zero and impose the condition that $\eta$ shall remain finite, we see that $\eta$ must approach a root of the limiting form of equation (14). This is 


$$
\sum_{i} e^{i} \psi_{i}(0) \eta^{m_{i}}=0
$$

the sum including only such terms as had the minimum exponent of $t$ in (14). In general this equation will consist of only one term, and hence its only solution is $\eta=0$. If now we wish to exclude the case where $\eta$ approaches zero with $t$, we must choose $c$ and $d$ so that two or more exponents of $t$ in (14) are equal to each other and less than any others. Let $\lambda=c / d$. Then the exponent $c m_{i}+d i$ becomes $d\left(\lambda m_{i}+i\right)$. As $d$ is independent of $i$, the exponent is a minimum when $\lambda m_{i}+i$ is a minimum. For small values of $\lambda, \lambda m_{0}<1$, and hence the exponent of the first term is less than any other;

$$
\lambda m_{0}<i+\lambda m_{i} \quad(i=1,2, \cdots, r) .
$$

Let $\lambda_{1}$ be the smallest value of $\lambda$ for which this is not true, i. e., for which there is an $i$ such that

$$
\lambda m_{0}=i+\lambda m_{i} .
$$

Let this value of $i$ be $i_{1}$, or if it happens simultaneously for two or more values of $i$, let $i_{1}$ be the greatest of these. Then it is easy to show that for increasing values of $\lambda$, the exponent $d\left(i_{1}+\lambda m_{i_{1}}\right)$ becomes and remains the minimum until it becomes equal to the exponent of some later term,

$$
i_{1}+\lambda m_{i_{1}}=i_{2}+\lambda m_{i_{2}} \leqq i+\lambda m_{i}
$$

where $i_{2}>i_{1}$. If there are two or more possible values of $i_{2}$, then $i_{2}$ is to indicate the largest of these. In like manner $d\left(i_{2}+\lambda m_{i_{2}}\right)$ becomes and remains the minimum exponent until it becomes equal to some exponent where $i>i_{2}$.

We proceed in this way, determining all possible values of $\lambda$ (not more than $r$ in number) for which $\eta$ does not become zero or infinite as $t$ approaches zero, but subject always to the condition that $i+\lambda m_{i} \leqq r$, as otherwise the term $\zeta e^{r} t^{d r}$ may become the principal infinitesimal, since we have no means of comparing the infinitesimals $\zeta$ and $t$. We can take account of this condition by setting $m_{r}$ arbitrarily equal to zero. If $m_{r}=0$, then $r+\lambda m_{r}(=r)$ appears in the equation for determining the last value of $\lambda$, and this value is to be treated like any previous one. If $m_{r} \neq 0$, then the coefficient of $\eta^{m_{r}}$ in (15) is zero and $\eta=0$ is a root. In this case there is a possible value of $\eta$ 
which approaches zero for all values of $\lambda$ for which we can determine its behavior with the assumptions that we have made. We cannot say whether or not it yields a solution unless we know the existence and values of derivatives of order greater than $(n+r)$.

If $\eta$ corresponds to an actual solution of (1), the identity of this solution is not affected by varying $\lambda$. Let us consider, then, the case that $\eta$ belonging to this solution becomes infinite for all values of $\lambda$. This means in particular that the coefficient of the highest power of $\eta$ in (14) approaches zero with $h$ when $\lambda$ has the values which make the exponent $\lambda m_{0}$ the minimum. This, however, shows that the coefficient of the highest power of $\eta$ involves a $\psi$ which is not $\psi_{0}$. If then we set $r=0$, this power of $\eta$ will not appear. But we know from Theorem 1 that setting $r=0$ gives the necessary condition for all solutions which approach $\left(x_{0}, y_{0}\right)$. As we are concerned only with these, the case that $\eta$ becomes infinite for all values of $\lambda$ is disposed of. If $\eta$ approaches zero for some values of $\lambda$ and becomes infinite for others, it is easy to show that there is an intermediate value of $\lambda$ for which it approaches a limit not zero.

Thus for any solution approaching $\left(x_{0}, y_{0}\right)$ whose character is determined by the assumed data, we have a certain value of $\lambda$ and an equation of the form (15) for determining the limiting value of $\eta$, or to write the equation more explicitly,

$$
e^{p} \psi_{p}(0) \eta^{m_{p}}+e^{q} \psi_{q}(0) \eta^{m_{q}}+\cdots+e^{s} \psi_{s}(0) \eta^{m_{s}}=0,
$$

$$
c m_{p}+d p=c m_{q}+d q=\cdots=c m_{s}+d s=\mu \leqq c m_{i}+d i .
$$

Or, since $\eta \neq 0$,

$$
e^{p-s} \psi_{p}(0) \eta^{m_{p}-m_{s}}+e^{q-s} \psi_{q}(0) \eta^{m_{q}-m_{s}}+\cdots+\psi_{s}(0)=0 .
$$

We now have that any possible solution is expressible in the form

where

$$
\begin{aligned}
& x=x_{0}+e t^{d}, \\
& y=y_{0}+e t^{d}\left(\alpha_{1}+\eta t^{c}\right)=y_{0}+a_{1} t^{d}+a_{2} t^{d+c}+\zeta_{2} t^{d+c},
\end{aligned}
$$

$$
a_{1}=e \alpha_{1}, \quad a_{2}=\lim _{t=0} e \eta, \quad \text { and } \quad \lim _{t=0} \zeta_{2}=0 ;
$$

and where $c, d, e$, and the limiting value of $\eta$, satisfy (17) and (18), and $e= \pm 1$. 
These are the necessary conditions for a solution. If, however, the limit of $\eta$ is a real simple root of (18), they are also sufficient. For let

Then

$$
y_{1}=y_{0}+a_{1} t^{d}+a_{2} t^{d+c} \text {. }
$$

$$
\begin{aligned}
F\left(x, y_{1}\right) & =h^{n}\left(\sum_{i=0}^{r} e^{i} \Psi_{i}\left(\eta t^{c}\right) \eta^{m_{i}} t^{o n_{i}+d i}+\zeta e^{r d r}\right) \\
& =t^{n d+\mu} \omega(t), \quad \text { where } \lim _{t=0} \omega(t)=0 .
\end{aligned}
$$

Developing $F_{01}(x, y)$ in the same form as (6), we get

$$
F_{01}(x, y)=h^{n-1}\left(\sum_{i=0}^{r} h^{i} \frac{\partial \phi_{n+i}}{\partial \alpha}+\zeta^{\prime} h^{r}\right)
$$

where $\zeta^{\prime}$ approaches zero with $h$. Substituting $\alpha=\alpha_{1}+\zeta_{1}$ in $\partial \phi_{n+i} / \partial \alpha$, we get

$$
\begin{aligned}
F_{01}(x, y) & =h^{n-1}\left(\sum_{i=0}^{r} h^{i} \zeta_{1}^{m_{2}-1} m_{i} \psi_{i}\left(\zeta_{1}\right)+\zeta^{\prime} h^{r}\right) \\
& =e^{n-1} t^{(n-1) d}\left(\sum_{i=0}^{r} e^{i} m_{i} \psi_{i}\left(\zeta_{1}\right) \eta^{m_{i}-1} t^{i d+\left(m_{i}-1\right) c}+\zeta^{\prime} e^{r} t^{r d}\right) \\
& =t^{(n-1) d+\mu-c} \Omega(t, \eta),
\end{aligned}
$$

where the limit of $\Omega(t, \eta)$ is the partial derivative of the expression in (16) and hence not equal to zero on the assumption that $\eta$ approaches a simple root. Since $\Omega$ is a continuous function of $t$ and $\eta$, we can find small intervals for $t$ and $\eta$ within which $|\Omega|>\epsilon$, where $\epsilon$ is some positive constant. If now

$$
y=y_{0}+a_{1} t^{d}+e \eta t^{d+c},
$$

$\eta$ having any fixed value in its interval, then

$$
\begin{gathered}
\Delta y=y-y_{1}=\left(e \eta-a_{2}\right) t^{d+c}=e \Delta \eta \cdot t^{d+c} . \\
F(x, y)-F\left(x, y_{1}\right)=\Delta y F_{01}\left(x, y_{1}+\theta \Delta y\right)=e \Delta \eta \cdot t^{n d+\mu} \Omega\left(t, e a_{2}+\theta \Delta \eta\right) \\
F(x, y)=t^{n d+\mu}\left[\omega(t)+e \Delta \eta \Omega\left(t, e a_{2}+\theta \Delta \eta\right)\right] .
\end{gathered}
$$

As $\omega$ approaches zero and $\Omega$ approaches some other limit, we can take $t$ so small that 


$$
|\omega|<|\Omega \cdot \Delta \eta|,
$$

and hence the sign of $F(x, y)$ depends on the sign of $\Delta \eta$. As in Theorem 2 , it now follows that $F(x, y)$ vanishes once and only once in the strip and hence there is a single valued function of $t$ which yields the complete solution belonging to this limiting value of $\eta$. A short computation will show that if we change the sign of $e$ and make no other change, we either get the same solution that we had before or else none at all.

All this is on the assumption that $\eta$ approaches a simple root of (18). If, however, $\eta$ approaches a multiple root, we substitute $\eta=e\left(a_{2}+\zeta_{2}\right)$ in (14) and arrange according to powers of $t$, up to $t^{d r}$. We then have an equation connecting $\zeta_{2}$ and $t$ of exactly the same form as equation (13) connecting $\zeta_{1}$ and $h$. With this we can proceed in the same way, getting $\zeta_{2}$ and $t$ expressed in powers of a new parameter as a necessary condition, which becomes sufficient if the equation for the new coefficient has a simple root. For a multiple root we must repeat the process. With regard to the continued repetition of the process, there are three possibilities: (1) it may come to an end at some point through the appearance of an equation for the next coefficient, which has only simple roots ; $(2)$ it may end on account of the non-existence of continuous partial derivatives of the orders required to carry it on ; or (3) the appearance of multiple roots may continue indefinitely. An example of this last is the case where $F(x, y)$ is such a function as $(y-\sin x)^{2}$. Here

$F(x, y)=x^{2}-2 x y+y^{2}-\frac{1}{3} x^{4}+\frac{1}{3} x^{3} y+\frac{2}{45} x^{6}-\frac{1}{6} x^{5} y+\cdots$,

and the coefficient of every term in the assumed solution will be a double root of the equation for determining it. Hence no finite number of terms from the development would enable us to determine the character of the solution, or assert that the origin is not a cusp, an isolated point, or a point common to two distinct branches.

The question of the derivatives of $\zeta_{1}$ with regard to $h, \eta$ with regard to $t$, etc., may be treated by expressing $x$ and $y$ in terms of these new variables, and finding the derivatives of $F(x, y)$ with regard to them, thus reducing the problem to that of Theorem 3.

HARVARD UNIVERSITY, September 1, 1909. 